\title{
DE FORMULARUM ORIGINE
}

\author{
SCRIPSIT
}

J. C. NABER.

Fides haberi solet $\left.{ }^{1}\right)$ Gaio docenti ${ }^{2}$ ) per lege(s) .... sublata(s) esse legis actiones, eo vel magis, quia concurrit Gellii testimonium $\left.{ }^{3}\right)$ : omn(emn) illa(m) duodecim tabularum antiquita(tem), nisi in legis actionibas centumviralium causarum ${ }^{4}$ ) lege Aebutia lata ${ }^{5}$ ) consopita(m). Ex diverso secundariae eiug sententiae: legibus illis effectum $\left.{ }^{6}\right) \ldots u t \ldots$ pr formulas litig(e)mus nemo fere hodie vult obtemperare ${ }^{7}$ ) ant ideo, quod non videatur sine formula apud iudicem constare posse, quid proprie in iudicium venire oporteat ${ }^{8}$ ), aut, quae sententia plures habet sectatores, propterea quod peregrinos diu ante legem Aebutiam petere potuisse

1) Gf. inprimis $W_{\mathrm{IASsak}}$, Prozessgesetze 1 (1888) p. 65/6: "Meines $W_{\text {issens lieg.t }}$ keinerlei Grund vor, die Richtigheit dieser Angabe zu bezteifeln." Soribebat quidem eodem tempore Soum, Institutionen (1888) p. 150: "kein Zaweifel, dass der alle Legisactionenprozess NICHT aufgehoben tourde."

2) IV $\$ 30$

3) XVI 10 \& 8 .

) Addit Gaius (IV $\$ 31$ ) dommi infeeti cansam.

5) Coniungit Gaius (IV $\$ 30$ ) duas Julias.

6) Prudenter observat Betumann-Hothweo, Civilprozess $\$ 55^{7}$ (II p. 6), non hoe dicere Gaium: formulas esse lege introductas, verba eius hanc quoque admittere interpretationem: abrogatis legis actionibus novas formulas in vacuum venisse. Etsi disputat contra WLASSAK, Prozessgesetze I p. 159 sqq., oui praeivit Erset.s, matcr. Grundlage der exceptio (1871) p. 32, p. 166/7. Rursus verum vidit Jöns, rön. Rechtswissenschaft I (1888) p. 176.

7) Soum, Institutionen (1888) p. $150^{5}$ : "dass die formula durch die lex Aehutia nicht exst eingefuht, sondern bereits zur Zeil der Legisaktionen aufgekommen isl, kamn. gegenwärtig als allgemein angenommen gelten."

8) Hoc argumento pugnat Huvelin, Mélanges Gérardisz (1907) p. 334 ; „il est... . évident qu'on ne peut se reposer sur les seules parties du soin de faire connaitre au juge sa mission etc." Quorsum igitur prisca litis contestatio (KELLER, Litiscontestation md. Uriheil $\$ 1)$ ? 
constat 1), quorum videtur 2) sacramentum irstum pronuntiari non potuisse ${ }^{3}$ ). Nam hoc disertis verbis docet orator in causa Caecinae ${ }^{4}$ ) et remota peregrinitate iam posse lege agi ${ }^{5}$ ); constat praeterea in iure cessionem, quae legis actionem imitatur $\left.{ }^{6}\right)$, propriam fuisse civium Romanorum 7). Ne illud quidem obstat, quod legi videtur apud Gaium ${ }^{8}$ ): epud praetorem.... peregrinum lege agi posse; etenim quis dubitat, quin sit apud magistratum populi Romani legis actio ${ }^{9}$ ? Sed non est nisi inter solos cives Romanos: apud Gaium ${ }^{10}$ ) utique eius condicionis litigatorem intelligendum satis hic demonstrat, quum doceat post sacramentum cundum ad Centumviros ${ }^{11}$ ). Excipienda quidem est repetundarum actionis cansa ex legibus Calpurnia et Iunia ${ }^{12}$ ), quibus licet quis dixerit ${ }^{13}$ ) actorem esse datum civem Romanum, qui pro

1) Tab. III 7. Cf. Momusen, ad legem de scribis (1843) p. 19/20.

7) Etiam Ludovico Mrters, Pripatrecht I (1908) p. 124 not. 45.

2) Exoipit Scrmiot, Ztschr. der Sav.-St. IX (1888) p. 142/43, Latinos et item Fisule, Abhandl. (1889) p. 69/70, foederatos. Neutri se valde repugnaturum olim edixerat KaHLowa, Legisactionen (1872) p. 3451 . Plane, quàm pro se laudat SchsidT, legem Lucerinam (Bruss, font. (1909) no. 104a) nullius in hanc rem esse momenti ipse demonstrat (p. 142; "es handell sich nicht um einen Process. . . mil cives Romani") et WLASSAK, Ztschr. git, XXVIII p. 121/2.

1) $33 \S 97$ (W t.as\$ak, Prozessgesetze II (1891) p. 186/7). Refragatur verbo tenus Desserteaux (Mél. Gúrardin (1907) p. 185/7), attamen adimit peregrinis ipse quoque omnem legis actionem, quae habeat verba "Ex ILнE QuIRITum" (p. 187 ima: "pérégrine elle ne pourra pas prouver qu'elle est libera ex iure Q."; of. ScImivt, Zlsehr. cit. IX (1888) p. 138 lin. 18/9).

5) Adnotat GInand, Hist. de l'organis. judic. I (1901) p. 216': "cela ne prouve pas que les pérégrins n'aient jamais la legis antio, mais cela prouve qu'ils ne b'ont pas toujours", quod pergit exsequi Ztschr. cit. XXIX (1908) p. 120 not. (Mélanges I. (1912) p. 119/20).

") Negare eopit quidem WLassak, Ztechr. eit. XXVI (1905) p. 403'; XXVIII (1907) p. 76 sqq., 83sqq. sed repugnat hactenus Miтtiss, Privalrecht I (1908) p. 276:4.

7) Hoc argumento pugnant Karlowi, Legisactionen (1872) p. $345^{1}$; W..CH apud Kellerua, Civilproz. (1883) not. 192 (pag. 56/7); Schmint, Ztscht. cit. IX (1888) p. $138 / 9$.

8) IV $\$ 31$.

9) Gai LI $\$ 24$.

10) IV $\$ 31$.

11) Cf. Whassak, Prozessges. I (1888) p. 204/5. Quo praterea pugnat argumeuto Bachofen, de Rom. iudicis (1840) p. 43, id quia refellit ipse Gains I \$ 193 , nos eo abstinemus.

12) L. repet. inscr. lin. 23. Cf. PEnnice, Lab. III p. 238: "es ist kaum müglich die Lücke anders. . . antszufüllen".

13) Dixit KI.ENze, fragm. legis Serv. p. XII. Cf. quoque SchmidT, Ztschr. der Sab.-St. IX (1888) p. 139; Whassak, Prozessges. II (1891) p. 186; Gmand, Ztschr. cit. XIV (1893) p. $44^{3}$ (= Mélanges I (1912) p. 104 not 2), 
peregrinis lege ageret 1 ), ipsam difficultatem non tollet. Manebit enim vel sic pro peregrino lege actum ${ }^{8}$ ). Praeterea videtur nulla esse causcl, quidni peregrini lege agere potuerint, de quibus speciebus postea formula in ias concepta sine fictione civitatis in eorum persona tenui', veluti si a re in personam fiat nomen transscriptitium ${ }^{3}$ ), si verbis fig.t obligatio praeterquam DARI SPONDES? - SPONDEO 1), consensuve 5); etenim, si recte habet formula: gi parer Dioni Hermaki filio Dafi OPOR'TERE, cur minus recte intendat Dio: AIO MLIH DARI OPORTERE ${ }^{6}$ )? Restant ea, de quibus' a peregrino lege agi non potest veluti de libertate vel de proprietate, agi tamen potest veluti de proprietate 7). Quamobren plerique coniciunt ${ }^{8}$ ) peregrinorum in usum formulas introductas esse diu ante legem Aebutiam et eas in factum conceptas 9); quo concess' requirunt ${ }^{10}$ ), ecqua causa praetorem cohibere potuerit, quominus inter cives perinde formulam daret sive consentientes sive novis exorientibus negotiis, de quibus nullae essent legis actiones proditae velut incerti stipnlationibus, emptionibus venditionibus, locationibus conductionibus, societatibus, mandatis ${ }^{11}$ ). Qui quum contendunt peregrinos ante legem

1) Cf. Cic, Accus. I $4 \$ 11, \$ 12 ; 20 \$ 65$ (legis izdiciique actorem).

2) Mitris, Privatrecht I (1907) p. 5230: "qusnahmsaeise." Ita nos quoqu's definivimus.

3) Gai III $\$ 138$.

+) Gai III $\$ 93$.

5) Hic non est vetus testimonium sed longe pretiosius Mauntri WLassak, Prozessges. II (1891) p. 148 not. 18 (i. f.). Superest quaestio, utrun ex eiusmodi contractn lege agi potuerit.

5) Causam vidit Aencrio-Rurz, le formule con demonstratio (1912) p. 49, sed non expressit.

7) Tab. III 7. De libertate idem esso ("wie... Eigenthum, so... Treiheit") probabiliter ducet sine teste Monmsen, Ges. Schr. TII (p. 10).

9) Nomina dabit Grrako, Hist. de l'organis.judic. I (1901) p. 216². Add. Bachofen, de Ron. iudiciis (1840) p. 117 (ia. p. 49).

9) WLASSAK, Prozessges. II p. 302 ima: "wenn nicht durchaus, doch überwiegend" : I p. 76: "durch die Auddehnung des Formalarverfuhrens vor dem Shalipraetor durfles: dan neben den honorarisohen die cinilen indicia entstanden seinz". Adstipulatur MitTets, Privatr. I (1908) p. 50/1. Ex diverso Arangio Rulz, le form. con demonstr. (1912; p. 69-72, fuisse putat formulas cum demonstratione.

10 ) Veluti Jöns, röm. Rechtswiss. I (1888) p. 179i80; WLassak, Prozessges. II (1891; p. 303 sqq.; CuQ, Instit. jurid. I (1904) p. 2866; M.tTtes, Privatr. I (1908) p. 46 sqq. Ex diverso docet LeNeL, Ztschr. cit. XXX (1909) p. 339-354, novas legis actioneE. permittendo praetorem subvenisse.

11) Nullas fuisse in eo genere legis actiones proditas, suspicatur W ,assak, Prozesszes. II (1891) p. 303 nut. 10 (i.f.), valde certus est MitTIs1s, Privatrecht I (1908) p. 50. Quippe quibus incertum petendum fuisset. Rebellat Arangio-Ruiz, le form. con demonstr. (1912) p. 55!: "non so vederne la ragione". 
Aebutiam non per legis actiones nisi in cansis exceptis sed per formulas litigavisse, aut non satis animadvertunt tertium dari: nullis conceptis verbis $\left.{ }^{1}\right)$ horum iudicia obstricta fuisse ${ }^{2}$ ) aut inique flagitant indicium aliquod ${ }^{3}$ ), ipsi quum ad libidinem iuris Romani memoriam a fundamentis renovent docendo non formulas sed iudicia legitima legis actionibus successiese. Quibus tunc corpus suum obiecit Moмmsen ${ }^{4}$ ), mihi vero nunc necessitas de formularum origine cum istis consistendi nulla 5), quia non secundariam tueri, sed principalem Gai sententiam convellere propositum est, quod mihi praestat ipse Graius, quam doceat ${ }^{6}$ ) formulas dari solitas ad legis action(is fictio)nem expr(essas 7), hoc, opinor, exemplo: ${ }_{n}$ i A. Agerius cum N. Negidio sacramento egisset hominem Stichum suum esse ex iure Quiritium ${ }^{8}$, tum si eius sacramentum iustum iudicari oporteret neque is homo arbitratu tuo a N. Negidio A. Agerio restituetur, quanti is homo erit, tanti condemna etc." Quid enim interest, talem praetor formulam det an legis actionem abroget? Consequens est non legibus sed iurisdictione legis actiones abrogatas 9 ). Nam, quod excogitavit Dernbora ${ }^{10}$ ): in quibusdam speciebus ${ }^{11}$ ) difficile

1) Concepta verha èt legis actionum sunt èt formularum.

1) Permiscet haee Schmid, Ztsehr. etc. IX (1888) p. 137/8.

3) Wlassak, Prozessges. II p. 302: "Keinē Spar einer dritten Prozessart (ist) erhalten", postquam docuerat ipse (II p. 216 summa) inter peregrinos nzunächst ohne Schriflformel" vertari solitum. Forte fortuna exstat vestigium: pro Mur. $11 \S 25$.

4) Ztschr. der Sav.St. XII p. 278.

s) Constitit GIaARD, Ztschr, cit. XIV p. $15^{8}$ (- Mulanges I p. 71 not, 2).

-) Gai. IV $\$ 10$ (quam emendavit D'A ulaing).

7) Non legis actionem ("die Prozessform"), sed intentionem ("die Klagbehauptung") in fictionem deduci, doctrina est Adotrm Wach apud Kectenum, Civilproz. (1883) not. $247 a$ (p. 96), quae sententia non convenit cum unico certo exemplo, quod habemus (Gai. IV \$ 32: si pignus captum esset).

3) Ad sententiam Avolru WACH requiritur fictio talis: "si A. Agorius hominem Stichum ex iure Q. suum esse in iure a $\mathrm{N}$. Negidio vindicasset". Interest quod ea non remittitur ipsum sacramentum.

9) Post legis antiones sublatas fictione opus non fuisse, quum fingi soleant abroganda, non abrogata, vidit quum Bloumans-Holsweg, Cioilproz. $\S 96$ ante notan 3 (II p. 305) tum Banon, Abhandl. I (1881) p. 213 ("erhielt der Magishral (durch Gesetze) das Recht der Formelconception", unod factum putat ( prozess (ist) durch Gesetze eingeführt worden") "so bedurfte es nicht der Fiction"). Sed hic nihil, ille ulterius nihil inde deducit, nisi, "dass diese also die aeltesten fictitiae actiones [des praetorischen Rechtes] sind".

10) Krit. Ztsehr. I (1858) p. 471.

11) "zoenn dem Kläger durch eine lex kein abstrakter Anspruch sondern nur das Recht zur Wornuhne einer legis actio zugetheill aar. So hatle der sponsor zunächst nur die manus iniectio wegen des depensums, der publicanus... die pignoris capio gegen des 
visum esse, legis actionis materiem et substantiam nulla fictione adhibita transfundere, id propterea non tenet, quia non tantum, quod ipsins doctrina postulat, legis actiones per manus iniectionem vel per pignoris capionem sed ceterae quoque praeter condictionem ${ }^{2}$ ) fictione adhibita transfusae sunt. Consequens est praeterea has formulas ad legis actionis fictionem expressas inter antiquissimas esse referendas ${ }^{2}$ ), iis quidem exceptis, quibus pecunia vel certa res debita petatur, quas eo tempore introductas, quo ceterae adhuc lites per legis actionem ordinarentur, sane est verisimile. De eo enim, quod nobis dari oportet, quum aut poterat sacramento agi aut per iudicis nudam postulationem, tertins quidam agendi modus introductus est ${ }^{3}$ ), qui aut fuerit oportet formula „8I PARET DARI OPORTERE" aut omnino libera nec ullis conceptis verbis constricta intentio. Nam, quum agitur de eo quod dari oportet, ress nullo modo ordinari potest, nisi ant actor dicat "AIO MIFI DARI OPO]TERE" aut magistratus dicat "BI PARET DARI OPORTERE" aut omnirlo libera peragatur intentio. Porro, si dixisset actor ${ }^{4}$ ): „AIO MIHI Darr OPORTERE" cetera aut sacramento expedienda fuerunt aut sine sacmimento, id est, per indicis arbitrive nudam postulationem; itaque restat aut formula ant omnino libera intentio, si quidem fuit tertius agendi modus, qua de re dubitari non potest. Formulam fuisse arbitratus est Keller ${ }^{5}$ ) de certa utique re, nam de certa pecunia actum putat per sponsionem et restipulationem tertiae partis. Quem hactenus refutavit EISELE ${ }^{6}$ ) hoc argumento, quod sponsione facta superest sponsionis summae, id est, certae pecuniae petitio et quaeritur agendi modns ${ }^{7}$ ).

Steuerpfichtigen". De pignoris capione valde contendit Wrassak, Prozessges. I (18843) p. 250 sqq.; de manus iniectione dubitat (ibid. p. 251 not. 22).

1) Gai IV \$ 33. Cf. Keller, Civilproz. not. 298 (p. 122).

2) Referendas vidit Keluer, Civilproz. \$25 (post notam 298): "(die) 7rigu" (mubv) doch gerude zu den Anfängen der Formelbildung mit gehören."

3) Gai. IV \$ 20 .

1) Dixisse putat KuRsow, Legisactionen (1872) p. 232.

s) Civilpraz. $\$ 18$ (post notan 243). Quidquid in contrarium disputavit KHüGer, process. Consuniption (1864) \$ 3 (p. 12/3), id feliciter refutavit Eısece, mater. Grunlluge der exceptio \{1871) p. 165/7. Sed, quo pugnat ipse (op. cit. p. 154-160): quod legis actio per condictionem tollat obligationem (Gai. IV \& 108) et quod idesu contingat legitimo indicio per condictionis formulam (Gai. IV $\$ 107$ ), ideo tieri, quia legitimum indicium per condictionis formulam substantiam intuentibus ( $n^{i n}$ Wesenllichen") sit legis actio per condictionem versaque vice, id Sextus Aelius rt fuerit probaturus metuo.

i) Die mater. Grundl. der exceptio (1871) p. 167-170. Ex diverso KEl.Lenus per omnia sequitur Bethmann-Holcweg, Civilprocess \& 44 post notam 17 (I p. 153/5).

7) Cf. praeterea Kruegen, process. consumption (1864) $\$ 3$ (p 12/3). 
Quocirca sumit ipse èt de certa pecunia è de certa re debita ante legem Aebutiam per formulam agi coepisse. Quod mihi quoque probabilius videtur quam per omnino liberam intentionem, etsi dubitari posse concedo. Ergo per legem Siliam et Calpurniam ${ }^{1}$ ) introducta proprie videtur formula ${ }^{2}$ ), quod potuit ignorare Gaius, si liber Aelianus non continebat nisi verba solemnia litigatorum. Potuit, inquam, pro legis actione habere ipsam condictionem, id est, denuntiationem, quae fiebat adversario ${ }^{3}$, ut ad iudicem capiendum die trigesimo adesset ${ }^{4}$ ). Quod ideo sanciendum fuit, ne scilicet occasione formulae periret reo legis Pinariae beneficium, qua cautum, ut post dies triginta daretur iudex quam postulatus esset 5), quod erat etiam in lege Siculorum Rupilia ${ }^{6}$ ). Sic intelligitur, cur ad condictionis fictionem nulla formula exprim(a)tur ${ }^{7}$ ); sufficiebat scilicet condictionem aut lege abrogari ${ }^{8}$ ) aut desuetudine obliterari, ut superesset formula 9). Intelligitur praeterea, quae res sollicitum habebat Gaium ${ }^{10}$ ), (quare) haec actio desiderata sit, id est, propter eandem causam, quapropter omnes legis actiones in odium venerant. Erat autem causa non ea, quam putat Gaius 11): quod nimia subtilitate veterum, qui tanc iura condiderunt, eo res perducta est, ut vel qui minimum errasset litem perderet, nam, quia iidem gratis praeibant, non poterat quisquam errando litem perdere, nisi stulte eorum consilia qui sprerisset. Fenerunt quidem legis actiones

1) Gai. IV \& 19.

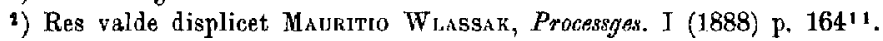

3) In iure, arbitror, etgi res controversa est. Cf. WACH apud Kellerus not. 242 (p. 91); Bетнmann.Hol.weg, Civilproz. \$ 44 post notam 9 (I p. 151/2); KarLown, Legisactionen (1872) p. $231 / 2$.

4) Gai. IV $\S 18$.

5) Gai. IV $\$ 15$. Formulae dationem et iudicis non utique coniunctan esse, vidit Lenes, Zischr. der Sao.-St. XXIV (1903) p. 337/8. Cf. WENGER, ibid. XXVI p. 529.

6) Accus. IV $15 \& 37$

7) Gai. IV \& 33 .

8) Putant quidam (cf. Whassak, Prozessges. I p. 129) legis actionem per condictionem et eam solam per legem Aebutiam esse sublatam. Propius verum hanc legis actionem solam perduravisse totam. Plane nihil obstat, quominus condictio, id est, denuntiatio lege Aebutia sublata sit, quum cetera legis actionam adminicula cnm ipsis legis actionibus sublata sint necesse sit iurisdictione, ut praediximus.

9) Hoc magis sensit quam expressit Kel.ek, Civilproz. $\$ 18$ post notam 247. Res mira ( $"$ schwer erklärbar") videtur A DOLPHO WACH not. $247 a$.

10) IV \& 20.

11) IV 830 . 
in odium, non tamen plebis sed praetorum, qui crescentibus in dies negotiis detenti bonas horas istis naeniis consumere gravabantur, proinde omnia moliti esse taedii finiendi cansa credendi sunt. Qui initio ad populum tulerunt leges vel ferendas curaverunt, deinde suo maste fietionibus se expediverunt, quam eandem ob causam institutum sst contra duodecim tabulas tacito consensu, ut litigantes non in iure apud praetorem manum consererent, sed ex iure manum consertum vocarent ${ }^{1}$ ). Summa haec est: condictionis formulas fortasse lege, reliquas omnes 2 ) a praetore ultro introductas esse ${ }^{3}$ ), non ut is consequeretur novam ${ }^{4}$ ) supplendi vel etiam corrigendi iuris civilis potestatem ${ }^{5}$ ), sed negoria celerius commodiusque dumtaxat conficiendi causa, proinde hane fuisse formularum originem et inter eives Romanos ${ }^{6}$ ). Potestas secuta est inventis (non) ex lege ${ }^{7}$ ) in factum actionibus ${ }^{8}$ ) et alterius generis fictitiis et exceptionibus ${ }^{9}$ ), quibus cogi posset iudex, ut res postulabat, timidus et verecundus ${ }^{10}$ ), contra ius civile absolvere vel condemnare. Qui prae-

1) Gell. XX $10 \$ 9$.

2) Quod scribit Gaius IV \& 11: complares actiones a praetore introductas es ie, id non pertinet ad verborum conceptiones, quas formulas appellamus, sed ad iura persequendi sive exigendi (cf. IV \& 1I2).

3) Itaque non est "irrig" (ErselE, mater, Grundl. der exceptio p. 32 not. 25a) sed potius richtig ;zu sagen die formulne entslammen dem praetorisehen Recht (z. B. MaYi:H, Comment. ad Gaium p. 47)."

1) Novam fuisse concedunt Puchta, Cursus der Institutionen \& 80. 3; Sонм, Insti.iu. tionen (1888) § 37. 7-9. Disputant contra Jörs, rön. Rechtswiss. (1888) p. 168/9; WLassak, Prozessges. II (I891) p. 351/3, qui quidem ipse se refellit (II p. 124, 126) sed fert auxilium ei LENEL, Ztschr. der Sav.St. XXX (1909) p. 329 sqq.

5) Hoe observavit recte Dewelius, Grünhul's Ztschr. XI p. 734.

6) Putat quidem Pernice, Lab. III p. 233/4, lege Silia datam esse condictionis actionem peregrino, multo vero magis lege Calpurnia, quam esse putat Calparniam. repetundarum.

7) Gai, IV § 109. Rehellat Eisecs, mater. Grindl. der exceptio (1871) p. $32:$ qiit, hieraus xich ergebende Macht des Praelors noss auf ainem Gesetze beruht habest." Quem refellit exemplum cancellarii apud Anglos.

B) In factum aetiones aliquanto post in ius conceptas inventas esse, sententia DemiLIt, Grünhat's Ztschr. XI p. 738 , est et vera. Contrarium docere coguntur, quibi1s videatur a iurisdictione iater peregrinos res descendere nec peregrino formula in ins concepta convenire. Quorum sunt Whassak, Prozegges, II p. 310 ima; I p. 76 ; Mitries Privatr. I p. 50/1.

9) Exceptiones post formulas inventas docet Gaius IV $\$ 108$. Ex diverso interdicta omnibus formulis antiquiora esse, communis est persuasio (Mitreis, Privalr. I (1908) p. 49: "unbestritlen").

10) De timiditate indicis est classious testis Gaius III $\$ 224$ (i. f.). Pauci fuerint oportat Galli Aquilii, quibus ad resistendum (pro P. Quinctio 9 \$33) quam doctrir.a 
toribus dicto audiens esse in castris manipularis vel centurio mature didicerat, ibidem praetor legatus legionis vel tribunus iudicem despicere ${ }^{1}$ ) et caloare; accedebat, quod vitio legis iudiciariae iudicibus plerumque solis vel paucis, cum praetura, quae dedisset, confligendum erat, quocirca mirari non debemus, cur Decemviri. ${ }^{2}$ ) Centumvirique ${ }^{3}$ ) dumtaxat numquam iugo colla submiserint fidnciam faciente quum numero tum quod non essent a praetore dati. Addit, Jöns ${ }^{4}$ ) populum ${ }^{5}$ ) pæssurum non fuisse; equidem rear, ne conaturum quidem fuisse praetorem ad hos iudices formulam dare, a quibus repulsam timere deberet, quum praesertim sine ullo periculo causam pertinentem lege ${ }^{6}$ ) ad Centumviros posset ad privatum iudicem et obnoxium sibi transferre. Sequitur, quando formulae pro legis actionibus in usum venerint, qui tractatus nobis non est coniunctus cum disputatione de tempore legis Aebutine, quandoquidem constare videtur lege neque abrogatas legis actiones neque introductas formulas. Est novissimum de ea re testimoninm Ciceronis anno 70 ius praetorium iam diu constitutum ${ }^{7}$ ), quod sine formulis ne cogitari quidem potest. Apertius etiam anno 76: sunt formulae de omnibus 8) rebus constitutae, ne quis aut in genere inimiae aut in ratione actionis errare possit. Lixpressae sunt enim ex unizucuinsque damno dolore incommodo calamitate iniuria publicae a praetore fommlae, ad quas privata lis accommodatur ${ }^{9}$ ). Decennio ante (anno 86) scripserat: in iure.... et exceptiones postulantur.... et ommis conceptio privatorum iudiciorum constituitur $\left.{ }^{10}\right)$, quorum neutrum convenit legis actionibus, utrumque formulis. Itaque, sicut Gaius, ita Cicero vidit quidem legis actiones,

tam ingenium tum divitiae animum facerent. Sed erant senatores xpцré tür rateinrwr.

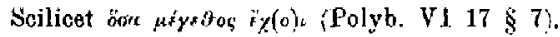

1) Arrogantiae certum indicium, quod in interdicto unde ve nobilis magistratus nobili cum praedone precative loquitur ("Restrons"), idem cun iudice numquam.

3) pro Caec. $33 \leqslant 97$.

3) Gai. IV $\$ 31$.

1) Röm. Rechtwoiss. (1888) p. 183.

s) "(die) öffentlich(e) Meinung."

6) Gio., de lege agr. II 17 \$4: pos volu(istis) de privalis hereditatibus Centumviros iadicare. Hoe postea coarotatum ad inofficiosi querelam, fortasse lege lulia, dictum est cap. harum observ. LXXVII.

7) Accus. III $44 \$ 114$

a) Quae quidem frequenter accidere soleant. De iis enim rebus, quae raro acoidunt, non semper sunt iulisi(a) prodil(a) (1. 1 D. 19. 5). Quod observat recte ScHots, das Gewöhren des Rechts.sehutzes (1903) p. 47.

9) Pro Q. Roscio $8 \$ 24$.

10) De invent. $1119 \$ 58$. 
sed praeterquam in hereditatem ${ }^{1}$ ) et in libertatem ${ }^{2}$ ) nisi in libris fortacise numquam ${ }^{3}$ ), quia in reliquis causis ipso adolescente loco iam cesserant formulis ${ }^{4}$ ). Quocirca fide dignus videtur, ubi inducit ${ }^{5}$ ) Crassum oratorem anno 91 de vet(ere) atque usitata exceptio(ne) cuIUs Pecuniae diES FUISse' disputantem, cui quidem exceptioni locus nusquam est, nisi in formulis quibus incertum petimus ${ }^{6}$ ). Quibus vetustior certae pecuniae formula, quod vidit BRONs ${ }^{7}$ ), comparet in lege Latina tabulne Bantinaes) (lin. 9/10: praetor recuperatores... dato iubetoque eum, sei ita pariat, condemnari), quam legem adseribunt harum rerum periti ${ }^{9}$ ) propter seripturae genus temporibus fere Gracchorum; ulterius demonstrare conatus sum ${ }^{10}$ ) esse Aciliae legis repetundarum particulam, proinde latam ante Chr, anjo 123 vel 122. Fuit hac diu litis formulariae monurnentim antiquissimum; esse desiit, postquam aliquanto vetustius ${ }^{11}$ ) testimonium ${ }^{12}$ ) inter senatus consulta demonstravit PARTSCH ${ }^{13}$ ), sed quod non pertineat ad

1) Acous. III $45 \$ 115$; de Örat. I $38 \$ 175$.

2) De domo $29 \$ 78$. Cf. pro Caec. $33 \& 97$.

j) Veluti debet ex libris hausisse totam materiem atque substantiam orationis pro Murena; indidem potest cognovisse actionem OPE. CONSIlloque tuo Furtum aro factum esse (de Nat. deor. III $30 \S 74$ ). Praeterea lege agendi vacabulum saepe usurpat de quolibet agendi genere (Accus. I 5 \$ 19; IV 16 \$39; pro Mur. $11 \S \approx 5$ ) quod fecisse videtur (KübLer, Ztschr. der Sab.-St. XXX p. 427: potest) etiam de Orat. $136 \S$ 167. Sed, etsi non fecit, nihil ad rem, quia res acta fingitur ante Hypsaeum consulem ( $\$ 166)$, id est, viginti minimum annis ante Tullium natum. (Rem valde amplificat. WLASSAK, Prozesigesetze I (1888) § 9).

4) Nonne igitur legis actio per manus iniectionem perseverat lege Ursoner.si (c. 61)? Primum nescio, quia interest inter manus iniectionem et legis actionrm per manus iniectionem sicut inter pignoris capionem ot legis actionem per pignouis capionem (WLassak, Prozesszer. I p. 255, qui eonvertit: „wie bei der manus inieot:a, so auch bei der pignoris capio") et inter sacramentum et legis actionem per sacramentum (Wlassar, op, cit, II p. 188 summa). Deinde videtur lex ad publicom dumtaxat coloniae eausam pertinere (WLassak, op. cit. I p. 92).

5) de Orat. I $37 \$ 168$.

) Gai. IV \& 131 (i. f).

') Kl. Schr. I p. 336. Cf. Girard, Ztschr. der Sav.St. XXIX (1908) p. 153 (= Mélanges I (1912) p. 152).

a) Bruns, font. (1909) no. 9.

9) Monmsen et Ritschl, quorum sententias exscripsit Maschke, zur Theorie und Gesch. der räm. Agrarges. (1906) p. 84.

10) Verslagen en mededealingen (Amsterdam) $4 \mathrm{X}$ (1909) p. 104 sqq.

11) Inter annos 190-160, si verum vidit PaRTsch, die Schriftformel etc. (1905)

p. 51. Cf. Giraho, Mél. Gérardin (1907) p. 256 not. 2 (p. 257).

12) Dittene., Syll. (1901) 928, 47-64.

15) Op. ait. p. 28 sqq. Erravit quidem, quum verteret (p. $30 \mathrm{ima}$ ) eis rovirons xais hóyors: in ea verba (= in e\&m formulam). Quod observavit Hitzig, Ztsohr. der Sab.-sit. 
cives Romanos. Quamobrem nondum iacet 1) GIRARD, cuius est opinio 2) post annum ante Chr. 149 legis actiones abrogari coepisse, hoc argumento, quod legis actiones profecto non ante abrogari coeperunt quam introduci desierunt. Esse autem introductam de pecuniis repetundis legis actionem sacramento lege Calpurnia ante Chr. 149 vel certe lege Iunia inter 149 et 123 constat ${ }^{3}$ ). Sed hoc argumentum obtinebit, si fuit lege Calpurnia iudicium privatum; alioquin, si publicum fuit 4 ), eandem ob causam hic legis actionem introductam esse dicemus ${ }^{5}$ ), quam propter conservata videtur litibus decemviralibus atque centumviralibus: quod cautus prator, ubi periculum est repulsae, id est, ad non a se datum et multorum capitum iudicem, formulam non est daturus ${ }^{6}$ ). Et contendit quidem Giraro ${ }^{7}$ ) lege Calpurnia privatum fuisse iudicium, sed contendit sine teste. Quod praeterea vir clarissimus docet ${ }^{8}$ ): legom Aebutiam necessario post Cinciam rogatam esse, id est, post annum ante Chr. 204, ideo, quod abrogationem legie actionum praeverterit lex Vallia 9 , Valliam Furia, Furiam Cincia, quod argumentum acceptum refert CARoto Georgio Brons $\left.{ }^{10}\right)$, id quam sit fragile, videbit quisquis inventorem inspexerit ${ }^{21}$ ). Remanet, ut abroga-

XXVIII (1907) p. 252/3. Sed manet iniuriarum formulae velut simulacrum: $\pi$ s

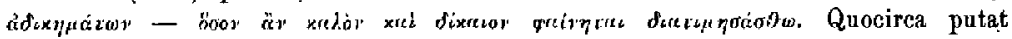
Annoro-Ruiz, le formule con demonstr. (1912) p. 62, iniuriarum actionem id temporis fuisse talem "quantum bonum et aequom tibi videbitur, iudex aestimato." Multo magis ita censet (p. 63/4) de formula depositi ceterisque, ubi postea fuit neius judex condemnato, si non paret absolvito."

1) Cf. ipsius defensio, ZZtschr. cit. XXIX p. 119-121 (Mélanges I (1912) p. 119-121).

2) Ztschr, ait. XIV j. 42-47 (Mélanges I. (1912) p. 101-107) alibique. Argumento it obviam Puchta, Cursus $\$ 80.9$.

3) L. repet. inscr. lin. 28 .

4) Ita sentit Mommsen, Strafrecht p. $190^{4}$.

5) Dixit post Maunitium W Lassak, Prazessges. I p. 135 (nder Praetor (kann) nur solchen Richtern Weisungen geben (= geben wollen),... die er ernannt.... hat"), Mitreis, Privatr. I (1908) p. 124"5: „Geschtoorene,... . die er nicht ermennt, kann der Praelor auch nicht durch formula instruieren (= instruieren wollen)". Recalcitrabunt enim undique trati. Quocirca iure pergit p. 5230: "tor einem (= an iinen) ständigen Gerichtshof hat es nie Formeln gegeben." It obviam Gunaro, Zlschr. cit. XXIX (1908) p. $126(=$ Mélangé I. (1912) p. 125/6) sed podissequae dumtaxat sententiae.

6) Dictnm est supra.

7) Ztschr. oit. XIV p. 45 lin. penult. (Mélanges I (1912) p. 105).

8) Ibid. (Ztschr, cit. XIV) p. 41/2 (= Mélanges I (1912) p. 100/1).

9) Valliam latam, quum legis actiones in usu erant, constat Gai testimonio (IV \$ 25).

10) $K l$. Schr. II p. $3(19 / 10$.

1) Tota res nititir eo, quod lex Furia excipit sex gradns cognatorum (Vat. fr. 301) Cincia quinque (ibid. $\$ 298,299$ ). 
tionem necessario praeverterit ius Aelianum, quasi non potuerit I.ripertitorum auctor post coeptas abrogari legis actiones, reliquas, quae in usu mansissent 1 ), ita tractare, ut formulas praeteriret, aut rursus Pomponius, si ille formulas in partem operis recepisset, in Tripertitorum descriptione receptas non commemorare 2 ). Quid quod addidit quaedam genera agendi quae deerant ${ }^{3}$ )? Potest idem auctor omisisse, quae exoleverant. Ergo revera nihil obstat, quominus aliquanto ante annum ante Chr. 200 legis actiones abrogari coeperint, et quominus, quod factum esse dicebamus ${ }^{4}$ ), formula Serviana auctorem habent urbanum praetorem ante Chr. 187. Similiter nulla causa est, quilni ante annum 200 formulae empti venditi locati conducti pro socio ceteraeque similes extiterint 5 ), quum praesertim satis constet venditi arbitrium Sexto Aelio fuise notum 6). Superest quaestio, utrum praetor formulas ipse invenerit an aliunde sumpserit. Et quidem apud solum populum Romanum et post Normannorum adventum apud Anglos formulis litigatum esse, esse videtur persuasio paene omnium commuris. Etsi de Graecis iam cogitare coepit W LASSAK $^{7}$ ); quin sibi vidori significavit Girard ${ }^{8}$ ), formnlas Romanas descendere a dicis Siculis, dicas Siculas ab instituto quodam Graecorum ${ }^{9}$ ). Cuius sententiae secunda pars nulli dubitationi subiecta est; fit enim dicarum scriptio obviam apud omnes Graeciae populos, quos novimus, velut Athenienses ${ }^{10}$ ), Delphos 11), Lesbios 12), Ilienses ${ }^{13}$ ), Teios ${ }^{14}$ ), Milesios ${ }^{15}$ ),

1) Pars manebat temporibus Gai (IV $\$ 31$ ).

2) Ea ratiocinatio est Paulr F'ridericr, Ztschr. cit. XIV p. 40/1 (Mélantges I. (1912) p. 99/100): "il reste en tout rass malatsé d'admettre que la section.. relolive à la procédure ent pu étre désignée comme relutive aux legis actiones, s'il eất existé à cóté d'eiles une autre procédure que l'auteur ext nécessairement expliquée lì."

1) L. $2 \S 7$ D. 1.2 .

4) Capite harum observ. LXXXIX.

5) Ea cura angit Lunovicum Mitreas, Privatrecht I p. 44/5.

B) L. $38 \$ 1$ D. 19. 1. Cf. MitTeIS, op. cit. p. 4832 .

7) Prozessges. II (1891) p. 301.

*) Mamuel (1911) p. 997 '. Adstipulatur Friniaux, Nowv. rev. histor. 33 (1909) p. 54!'3.

9) "zuse pratique hellénique."

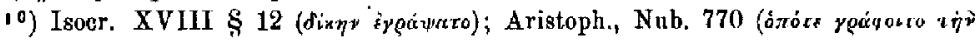

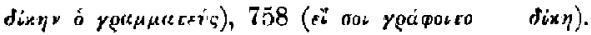

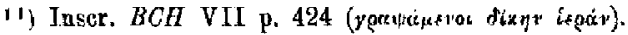

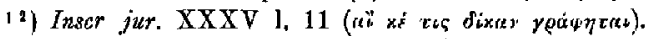

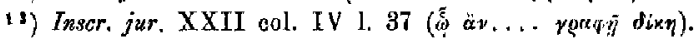

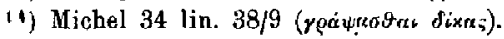

. $\left.{ }^{6}\right)$ Inser. Mileti III (Delphin.) 33 (c), 11; 35 (e), 10/1;147, 30/1;147,34/5;1:0,

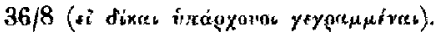


Macedones Aegypti dominos 1), atque, unde disputatio profecti est, Siculos 2). Et solet ferme dica adversario seribi ab actore, scripta porrigi magistratui eiusve scribae et ab hoc in volumina publica transscribi, quod utrumque apud Athenienses medio genere dicitur yuxidens $4\left(x,{ }^{3}\right)$,

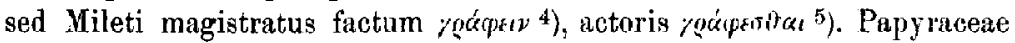
quoque dicae seribebantur ${ }^{6}$ ) adversario ab actore ${ }^{7}$ ), aliquando ${ }^{2}$ tô

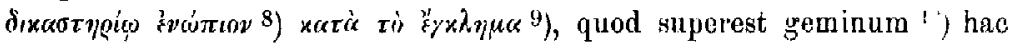

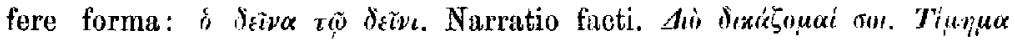

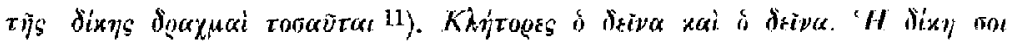

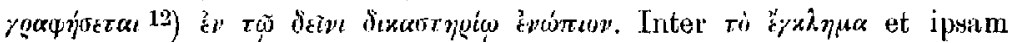

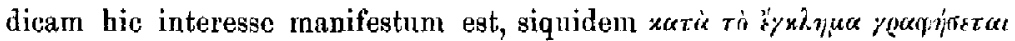
i $\delta i x \eta$; sed videtur sic distingui posse ibi tantum, ubi in iure scribenda dica est, postquam iisdem fore ${ }^{13}$ ) verbis domum denuntiatum sit;

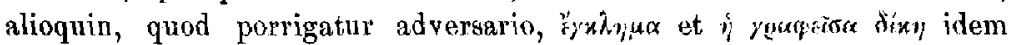
futurum 14). Ergo iam constat dicas Siculas a communi Graecorum

1) Veluti Pap. Lille 29 I 2 (dixyr rerequissos); Pap. Petrie TIT 21 (a)-(f), 21 (g) lin. 12, lin. 32; Pap. Hibeh 30, 24/5; Pap. Hal, 1. passin (pag. 2\$L).

2) Accus. IV $14 \$ 87$ (scribitur Heraclio dica); $17 \$ 42$ (iulset. . citavi Hemolium et eas qui dicam seripsernat); $24 \$ 59$ (postulant, nt...ex lege Rapilion dicam seribi iubeat.

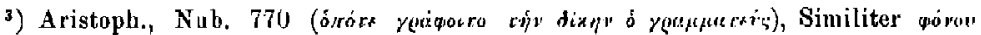

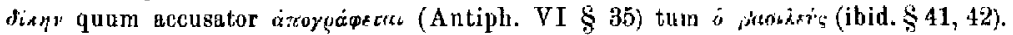

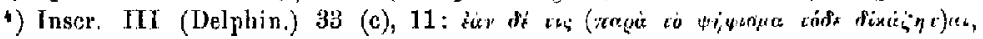

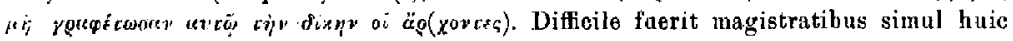
praecepto obtemperare et pedisseguo (1in. 12): ( Suppletum (pro , "ydi) oportuit : alida.

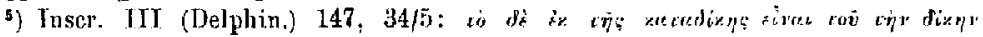
yeretiontitror.

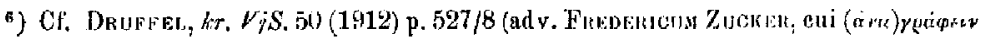
est $=$ roorstiver).

7) Pap. Petrie I[I 21 (a)-(f). His, ut vidletur, non inspectis seribi doeet Fils l, Uux, Hotw. rev. histor. XXXIII (1909) p. 543 (ia. p. 541), "par le greffer."

B) P. Petrie III 21 (g), 32/34 (Chrestom II 21); F. Hibeh 30, 24/5.

2) P. Petrie III 21 (g), 12; III 21 (c), 9/10 (Chreston II 28).

40) P. Petrie III 21 (g), $12-36 ;$ P. Hibeh $\$ 0$.

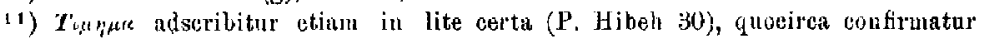

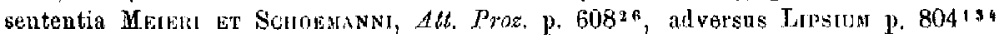
Att. Reght p. $822^{\text {s". }}$

12) Alterum exemplar (P. Hibeh 30) habet asroperstiom

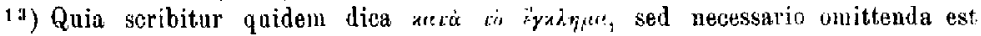

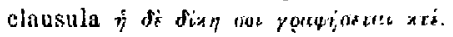

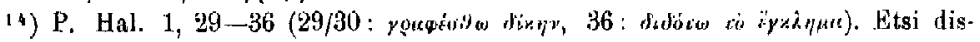

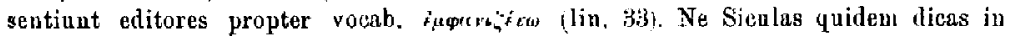
inre seribi solitas verisimite est (Funiatx, Nout. rev. histor. 33 (1909) p. 585/4!!]. 
instituto descendere; restat altera pars Girardi sententiae: a disis Siculis vel Graecis formulas descendere Romanas. Sed antequam ad propositam venio quaestionem, e re esse videtur sub uno conspectu ponere, quee sint communia diearum et formularum, quae intersist, ut omni errore sublato rem plane, quae in disceptationem veniat, videre possimus. Par causa est hactenus, quatenus et dica et formila scribitur et utraque continet causae coniectionem ${ }^{1)}$ et eam prinipalem 2). Sed in eo dispar, quod formula est, dica non est, epistula pruetoris ad iudicem, qua causae coniectio includitur et quasi continetur ${ }^{3}$ ). Ergo dica non est formula, sed potest facillime in formulam verti exemplar magistratui porrectum subscriptione eius, qualem habent

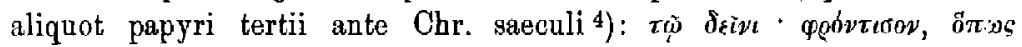

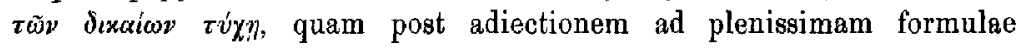
speciem nihil desit ${ }^{5}$ ). Nam plane ad rem non facit ${ }^{6}$ ), quod P. Petjie cit. ${ }^{7}$ ) is cui scriptum erat pQonc

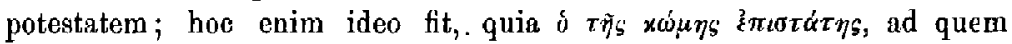
subscriptione strategi causa mittebatur, non poterat instam ferre sententiam, sed tantummodo renm hortari, ne pergeret actoris postulationi inique resistere, quocirea usitatior est subscriptio: $\mu \dot{\alpha} \lambda \iota \sigma \tau \alpha$ dúxhvoov

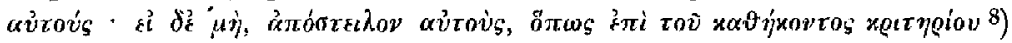
$\left.\delta \iota \alpha \times \operatorname{co} \vartheta \tilde{\omega} \sigma \omega \nu^{9}\right)$. Ne. strategus quidem iudieare solet ${ }^{10}$ ) sed ab epistata

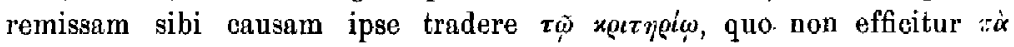

1) Eisece, mater. Grundl. der exceptio (1871) p. 172: "die formula (enthiell schon) einen toesentlichen Theil der causae coniectio."

2) Gukno, Manuel (1911) p. 996, p. 997': "(le) programme." Non recte divit (p. 996) formulam, quas iudisi detur, a pratore concipiendam ("qui... rédige"). Nam hoc actorem spectat, non praetorem (cf. e. g. Collat. II $6 \S 1$, $\$$ व; Gai, III § 224; IV $\$ 68$ (i. f.I; Lener, Ztachr. der Sav,-St. XV (1894) p. 388/9; quem refutare conatur Sснотт, dos Gewähren des Rechlsschutzes (1903) p. $49 / 50$.

3) Cf. CvQ, Tustit. jurid I (1904) p. $285^{3}$.

4) Pap. Magd. 4; 25; 34. Respicitur par species: Pap. Petrie II 2 (2) lin. 2 (Göll. gel. Anz. 1895 p. 146).

b) Putat quidem Koschaken, $G G A 169^{\circ}$ (1907) p. 818 (ad $B Q U$ 136, 24-2'), contra naturam esse formulae (

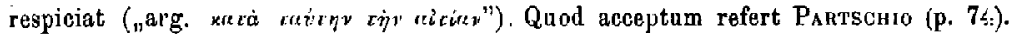

6) Quia non, ut Bousaro, les instrwetions berites etc. (1906) p. 96/7 (adnot.) de vi ac potestate $c \hat{\eta} ; \dot{i}$ royorep $\tilde{\eta} s$ quaerimus, sed tantummodo de verborum tenore.

т) II 2 (2).

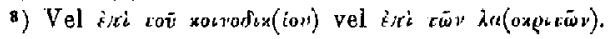

9) Pap. Magd. passim. Of. Pap. Hamb. 25.

10) Tertio ante Chr. saeculo. 
xptrỵgux esse in potestate strategi 1 ). Nam ne Centumviri quidem in potestate praetoris, etiamsi maxime, quum ad Centumviros i(bi)tur, ante lege agitur sacramento apud praetorem 2), deinde ab hoc causa Centumvirio traditur. In potestate strategi ita iudicia forent, si constaret iudicare ea ex formula a stratego data ( gemäss der ihn(nen) vom axpar $\eta$ ròs ertheilten Vollmacht"), quod ponit pro certo l'aulus Meyer ${ }^{3}$ ) laudatque

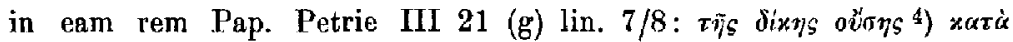

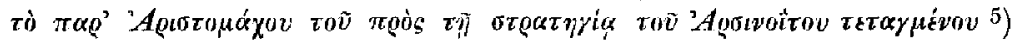

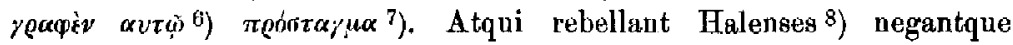

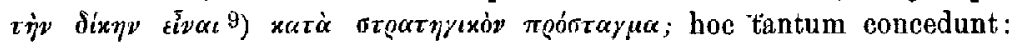

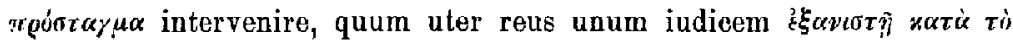

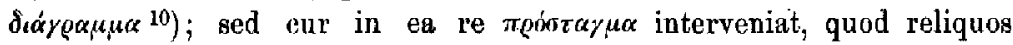
iudices ${ }^{11}$ ) iudicare iubeat, non $\tau \dot{o ̀ \nu} \xi^{\xi} \xi \alpha \nu \alpha \sigma \tau \alpha 9 \dot{\nu} \nu \tau \alpha$ retet, facile dietu non fuerit, dicere denique Halenses conati non sunt, sed tantummodo rem quaestiones permiscendo ${ }^{12}$ ) complicare. Iam ad Siculas dicas quod uttinet, potest coniectura capi datis lege Rupilia indicibus cum sub-

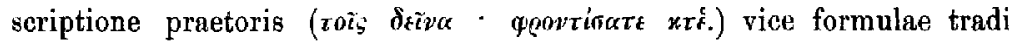
solitas; apud Athenienses autem hoc modo litigatum esse, tantum non

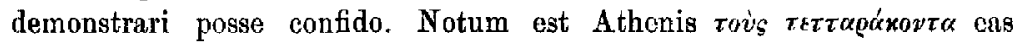

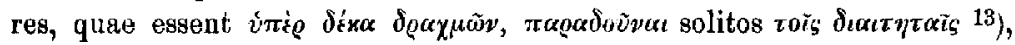

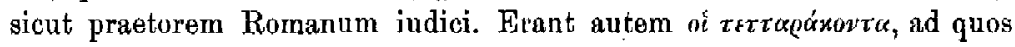
in ius adeundum erat omnibus de rebus, quibus proprius iudex con-

1) Putat DrtfFres, lir. TjS. 50 (19L2) p. 529/30, eatenus in potestate strategi esse indicia, quatenus a stratego causae its tradantur.

2) Gai. IV \& 31 .

) Klio VI p. $461 / 2$.

Sub proedro.

5) 1. e. ab ipso stratego. Cf. Dikaiomata p. $206^{4}$.

8) Interpretantur Halenses (Dikaiom. p, 206): fix' av่rō, rectius, ni fallor, Mever, Mitreis alii: roós avióv. Cf. Pap. Taur. 1 IV 24/5, quo remittit Druffel, kr. $V_{j S .}$ 50 (1912) p. 525. Plane, si acpos «v̀ cóv interpretamur, non erit, quo referatur.

7) Si verum vidit MEYen, vertendum erit formula.

") Dikaiom. pag. 209.

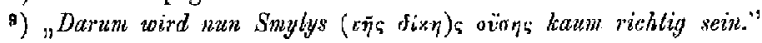

10) Eo pertinet non tantum F. Petrie III 21 (g), 7-11, sed etiam P. Hal. 9.

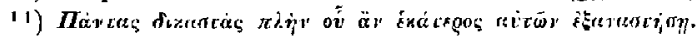

12) "Nur wenn eine der Parteien einen der Richter ablehnen wollte, musste sie sich... an den Strategen toendin, der dant in seinem rogoorcyus sich aussehliesshich auf DLESE Frage der Besetzung der (= der übrigen) Rishterstühle bexhrüglbte". Evanescet ortificium, si quis pro diese reposuerit die, quod sensus postulat. Nam non est eadem quaestio (Frage), sed diversa.

13) Aristot., resp. A.then. 53 \& 1. 1bid. 58 \& 2 habet árodiüru. 
stitutus non esset 1). Et scribunt Meier et Schormann ${ }^{2}$ ), nec quidquim ibi mutat Lipsius: nihil ultra quam ${ }^{3}$ ) $\pi$ cocedoviva Atheniensem magistratum arbitro. Quod inprimis mirificum est; dicendum enim potius

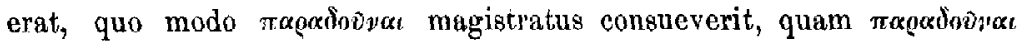
solitum nec ultra. Atqui veram quaestionem ne HudTwalcker quidem attigisse videtur ${ }^{4}$ ), attigit HUBlen' ${ }^{5}$ ) itaque definivit: consuesse magistratum libellum accusatorium, quem ab accusatore accep(isset) - intel-

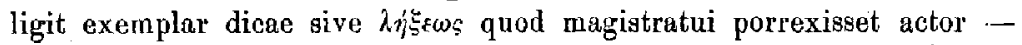
eum igitur libellum magistratum consuesse arbitro trad(ere), quanquam testem laudat nullum. Potest autem rei cognosci veritas ex oratione adversus Callippum $\left.{ }^{6}\right)$, ubi hic legitur ab arbitro abiisse $\alpha v(\varepsilon) \lambda(\dot{\alpha} \mu \varepsilon v(s)$

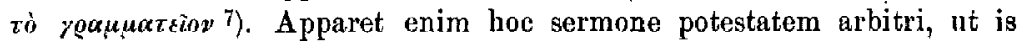

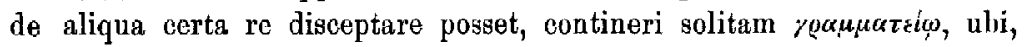
quia dabatur arbiter a magistratu certae finiendae controversiae, scriptum fuisse consentaneum est, de ea re ut iudicaret, mandatum, quod a formula iuris Romani nibil differt. Ergo recte, ambigue licet, vertebat Wolf actionem, nam formula quoque hac appellatione saepe censetur, non recte in adnotatione Reiske libellum accusationis, quia dis:n sive $\lambda_{i} \xi \iota s$ a litigatore porrigitur, requiritur autem, quod praetorem

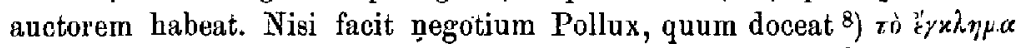

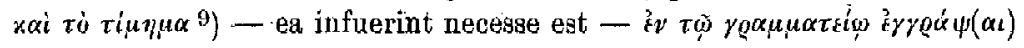
non magistratum, sed $x(b y)$ xolyovt( $\alpha)$, id est, agentem. Quae sententia, si habet recte, iam nihil interfuit inter $\tau \dot{\eta} \nu \hat{\lambda} \tilde{\eta}_{t} \nu$, quam magistratui

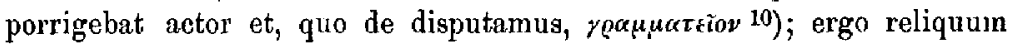
esse videtur, quod supra placere diximus Huberto: ut illam actor

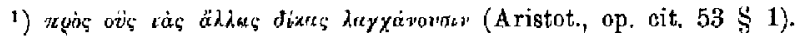

2) Attiseher Prozess p. 623/4 (Lipsuss p. 825).

3) Nihil ulira quam nune delet (Att. Recht p: 835).

5) Frustra praeterea a me inspectus est H. M. E. MEYER, die Pribatschiedsrichte' und die oeffentl. Diaeteten Athens (1846).

5) De arbitris atticis (1885) p. 42 .

6) Demosth. $52 \S 14$.

7) Similiter apud magistratum qui desistere velit, ei necesse est inde (Demostl.

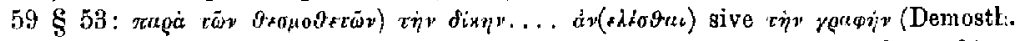
$59 \$ 53, \S 69 ; 58 \S 32$; Dinarch. I $\$ 94)$. Esse idem et. non.idem, declarabitur

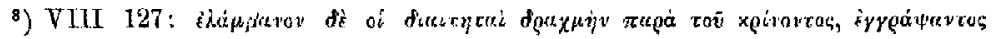

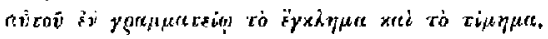

9) Latine vertam: intentionem el condemnationem. Est interdum ipxinjer idem atqu3. dica (Demosth. $34 \$ 16 ; 38 \$ 15$ ) sed non semper (Demosth. $25 \$ 55$ : «̋

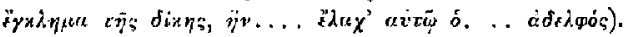

10) Ita sentit Lipsivs, Att. Recht p. $842^{4 a}$.
} statim. 
magistratui, hic deinde arbitro porrexerit, atque ita non tantum libelli ea, sed etiam, quod alibi ${ }^{1}$ ) suadere cupit Pollux, formulae vice functa sit. Nisi non est verisimile in civitate literatissima a magistratu libellum ad alium iuris usum translatum esse neque id compendiosa licet subscriptione declaratum, qua cum adiectione libellus iam nihil distat a formula. Ergo, si quid aliud, videntur formulas e iure Graeco, Attico praesertim, Romani mutuati esse. Licet autem èt Athenis quarto ante Chr. saeculo èt aliquanto post Romae per formulas litigatum sit, hoc tamen interest, quod Attici magistratus formulis numquam usi sunt tamquam instrumento quodam, quo iudices datos cogerent ius civile deserere eaque institnta amplecti, quae signassent ipsi. Quocirca Athenis nullum extitit ius honorarium uno, ni fallor, capite excepto quod indicavimus olim ${ }^{2}$ ) nec iterabimus.

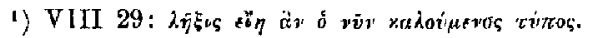

2) Observ. cap. CVI. 